\title{
Rauchstopp reduziert das Rezidivrisiko
}

\section{Hier steht eine Anzeige.}

Fragestellung: Wird durch das Einstellen des Rauchens bei Patienten, die eine transitorische ischämische Attacke (TIA) oder einen Schlaganfall erlitten haben, das Rezidivrisiko reduziert?

Hintergrund: Rauchen ist ein wichtiger Risikofaktor für Herzinfarkt und Schlaganfall. In unterschiedlichen prospektiven Registern waren 12-37\% aller Patienten, die einen Schlaganfall erlitten, Raucher. Trotz der Empfehlungen aller nationalen und internationalen Leitlinien, nach einer TIA oder einem ischämischen Schlaganfall das Rauchen einzustellen, gibt es sehr wenig Daten, die belegen, dass die Einstellung des Nikotinkonsums tatsächlich das Schlaganfallrezidivrisiko reduziert.

Patienten und Methodik: Die Publikation stützt sich auf eine Auswertung der Studie IRIS (Insulin Resistance Intervention After Stroke), in der Patienten mit ischämischem Schlaganfall oder TIA zu einer Behandlung mit Pioglitazon oder Placebo randomisiert und über 4,8 Jahre nachverfolgt wurden. Zum Zeitpunkt des Studieneinschlusses und jeweils nach einem Jahr wurden die Patienten bezüglich ihres Tabakkonsums befragt. Der primäre Endpunkt der Analyse war ein erneuter Schlaganfall, Myokardinfarkt oder Tod. Im Rahmen eines Cox-Regressionsmodells wurde die Häufigkeit der vaskulären Endpunkte berechnet und für Alter, Geschlecht und die wesentlichen vaskulären Risikofaktoren korrigiert.

Ergebnisse: Zum Zeitpunkt des Indexereignisses (ischämischer Schlaganfall oder TIA) waren 1.072 Patienten Raucher (28\%). Zum Zeitpunkt der Randomisierung hatten 450 Patienten (42\%) mit dem Rauchen aufgehört. Das 5-Jahres-Risiko, einen Schlaganfall oder Myokardinfarkt zu erleiden oder zu versterben, betrug 15,7\% bei den Patienten, die mit dem Rauchen aufgehört hatten, und 22,6\% bei den Patienten, die weiterhin rauchten. Dies entspricht einer Hazard Ratio von 0,66, die signifikant war.

Epstein KA, Viscoli CM, Spence JD et al. IRIS Trial Investigators. Smoking cessation and outcome after ischemic stroke or TIA. Neurology 2017; 89: 1723-9
Schlussfolgerungen: Die Daten der vorliegenden Studien zeigen, dass die Beendigung des Tabakkonsums nach einem ischämischen Schlaganfall oder einer TIA über einen Zeitraum von 4,8 Jahren signifikant das Risiko reduziert, erneut ein vaskuläres Ereignis zu erleiden.

\section{- Kommentar von Hans-Christoph Diener, Essen}

\section{Höchster Effekt für Schlaganfall und Tod}

Die Subgruppenanalyse der IRIS-Studie ist eine der ersten größeren Studien, die den Einfluss des Rauchens auf das Rezidivrisiko eines ischämischen Schlaganfalls oder einer TIA untersucht. Betrachtete man die Untergruppen, war der therapeutische Effekt am höchsten für die Mortalität und den Schlaganfall. Die Rate an Myokardinfarkten wurde nicht signifikant reduziert. Leider ist es im klinischen Alltag nicht damit getan, Patienten aufzufordern, mit dem Nikotinkonsum aufzuhören. Ein anhaltender Erfolg ist meist nur zu erzielen, wenn die Patienten in entsprechende Rauchentwöhnungsprogramme eingeschlossen werden. Diese werden in Deutschland von vielen Krankenkassen angeboten. 